\title{
Research on Constant Force Polishing Method of Curved Mold Based on Position Adaptive Impedance Control
}

\section{Guangsheng Chang ( $\square$ changuansen@163.com )}

BJUT: Beijing University of Technology

\section{Ri Pan}

BJUT: Beijing University of Technology

\section{Yinhui Xie}

Chinese Academy of Science

Jinxing Yang

Chinese Academy of Science

\section{Yong Yang}

Chinese Academy of Science

Jun Li

Chinese Academy of Science

\section{Research Article}

Keywords: Adaptive impedance control, Curved surface mold polishing, Polishing simulation

Posted Date: February 24th, 2022

DOI: https://doi.org/10.21203/rs.3.rs-1382963/v1

License: (9) (i) This work is licensed under a Creative Commons Attribution 4.0 International License. Read Full License 


\title{
Research on Constant Force Polishing Method of Curved Mold Based on Position Adaptive Impedance Control
}

\author{
Guangsheng Chang ${ }^{1,2}$ Ri Pan ${ }^{1}$ Yinhui Xie ${ }^{2, *}$ Jinxing Yang ${ }^{2}$ Yong Yang ${ }^{2}$ Jun Li ${ }^{2}$ \\ 1. Beijing Key Laboratory of Advanced Manufacturing Technology, Beijing University of Technology, Beijing, \\ China
}

2. Quanzhou Institute of Equipment Manufacturing Haixi Institutes, Chinese Academy of Science, Quanzhou, China)

\begin{abstract}
The quality and life of the injected product is affected by curved mold surface quality, so the polishing of the mold is essential. Small polishing tool are controlled by constant displacement polishing in the conventional method. The force will vary when polishing the mold with small polishing tool, thus affecting the surface quality of the mold. The robot constant force polishing method is proposed to solve this problem, which is achieved by a position-based impedance control algorithm. The proposed method can produce continuous and stable force. The effect of the constant force control method on the surface quality is analyzed by simulating this paper, proving the correctness of the constant force control method. The initial roughness of the mold surface is $1.912 \mu \mathrm{m}$, the result is $1.419 \mu \mathrm{m}$ in the traditional method, however the result of constant force polishing is $0.807 \mu \mathrm{m}$. The percentage of the decrease in surface roughness is $25.78 \%$ in the conventional method, whereas the reduce percentage is $57.79 \%$ in the constant force control(CFCP) polishing method. The feasibility of the constant force controlled polishing method is verified.
\end{abstract}

Keywords : Adaptive impedance control; Curved surface mold polishing; Polishing simulation

\section{Introduction}

The molded products cannot be separated smoothly from the mold due to the poor surface quality of the mold. Mold surface quality will affect the manufacturing efficiency and working life of products, so it is extremely vital for workpiece to machine a high-quality mold surface. As pointed out in previous research, the contact force between the grinding tool and workpiece is the key factor that affects the polishing quality [1]. To realize the control of constant force, scholars have investigated machining methods from a variety of perspectives [2]. Huang et al.[5] developed the passive compliance tools (PCTs) to grind and polish the distorted vanes, and the PCTs with passive force control can maintain reasonably constant material removal. Mohammad et al. [6] proposed a force controllable end-effector for automatic deburring, which can reduce inertial effect of polishing motor and spindle driven by micro driver. Chen et al.[7] proposed an end-effector, including gravity compensation force controller and two new eddy current dampers, which were integrated into the end-effector to improve the dynamic performance of the system and suppress the vibration during grinding and polishing. Tang et al. [8] proposed a multi degree of freedom flexible terminal actuator for mold polishing robot. The buffer spring ensures the working pressure between the grinding head and the workpiece changes in a constant range.

However, the above constant force compensation devices possess the disadvantages of low

Guangsheng Chang(changuansen@163.com)

Ri Pan(panri@bjut.edu.cn)

Yinhui Xie*(xyh1932@fjirsm.ac.cn, *corresponding author)

Jinxing Yang(jxyang@fjirsm.ac.cn)

Yong Yang(yangyongzp@163.com)

JunLi(junli@fjirsm.ac.cn) 
precision, slow response and poor environmental adaptability. So many researches concentrate on active force control. Duan et al. [9] used the force closed-loop feedback control method to polish the rail so that the amplitude of force fluctuations decreased by more than $10 \%$. Tian et al. [10] proposed a robot polishing tool position and pose generation algorithm based on force-position-pose decoupling control, the surface quality of the workpiece is greatly improved. Dong et al.[11] provide a stable control system for pneumatic polishing to achieve reliable control of the polishing force and improve the surface quality of the workpiece. Ding et al.[12] put forward an adaptive proportionalintegral control algorithm for constant force polishing to ensure contact force accuracy for polishing systems with different stiffness.

The mentioned research demonstrates that active power control can replace passive force control and offers broader application prospects. Based on the above analysis, a constant force polishing method with position-based adaptive impedance control is proposed in this paper. The remainder of this paper is organized as follows: The shortcomings of traditional control polishing is analyzed, and the method of constant force control polishing is proposed by means of simulation in Section 1. A constant force control for robotic polishing using an impedance control algorithm is described in Section 2. The constant force control method is validated by mold polishing experiments in Section 3 .

\section{Analysis of control methods of robot polishing}

\subsection{Conventional robot polishing control method}

Small polishing tool are controlled to machine molds at constant displacement in robotic conventional polishing methods. At the same time, the small polishing tool is only assigned to a fixed polishing path without force feedback. The robot finishes the mold surface following a grating path with constant displacement control as shown in Fig. 1. The variation of the contact area between the small polishing tool and the mold and the fluctuation of the polishing force are affected by the change of the mold curvature.

Mold machining is affected by the force fluctuation, so it is hard to hold the stability of polishing accuracy. The effect of Constant Displacement Controlled Polishing (CDCP) on mold surface quality is investigated by simulation in the next section.

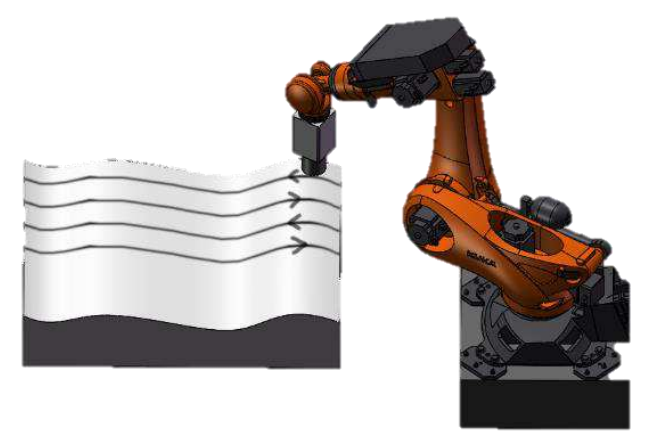

Fig. 1 Robot polishing process

1.2 Simulation analysis of constant displacement control polishing(CDCP)

The simulation is carried out to discover the influence laws of the curvature of the mold on the force fluctuation. The center of the mold is set as the coordinate origin, then the mold is loaded with $100 \mathrm{~N}$ by the small polishing tool, and the displacement (or deformation) of the small polishing tool is $8.6 \mu \mathrm{m}$. The displacement (or deformation) is $11.3 \mu \mathrm{m}$ when a load of $150 \mathrm{~N}$ is applied. The displacement (or deformation) corresponds to $13.6 \mu \mathrm{m}$ for a small polishing tool of $200 \mathrm{~N}$. The 
displacements of three sets of forces that match are shown in Tab. 1.

Tab. 1 The correspondence between the force and displacement of the small polishing tool

\begin{tabular}{ccc}
\hline $\begin{array}{c}\text { Experiment } \\
\text { Number }\end{array}$ & $\begin{array}{c}\text { Force } \\
(N)\end{array}$ & $\begin{array}{c}\text { Displacement } \\
(\mu m)\end{array}$ \\
\hline Group 1 & 100 & 8.6 \\
\hline Group 2 & 150 & 11.3 \\
\hline Group 3 & 200 & 13.6
\end{tabular}

Five different locations are selected in the mold, and the same displacement load of the small polishing tool is applied to the selected position of the mold respectively.

The simulation settings parameters are as follows:

1) The contact style between the small polishing tool and the mold is defined as frictionless contact, the normal stiffness factor is 10 [13].

2) Method of meshing is automatic: The whole size of the grid is $3 \mathrm{~mm}$. The local refinement to influence the ball radius is $2 \mathrm{~mm}$ and the unit size is $0.1 \mathrm{~mm}$.

3) The bottom of the mold is fixedly constrained. The load of the small polishing tool is applied vertically to the mold surface, while the tool is limited in other directions, which is similar to the actual machining situation. The simulation flowchart is shown in Fig. 2, where the model material parameters are given in Tab. 2 . 


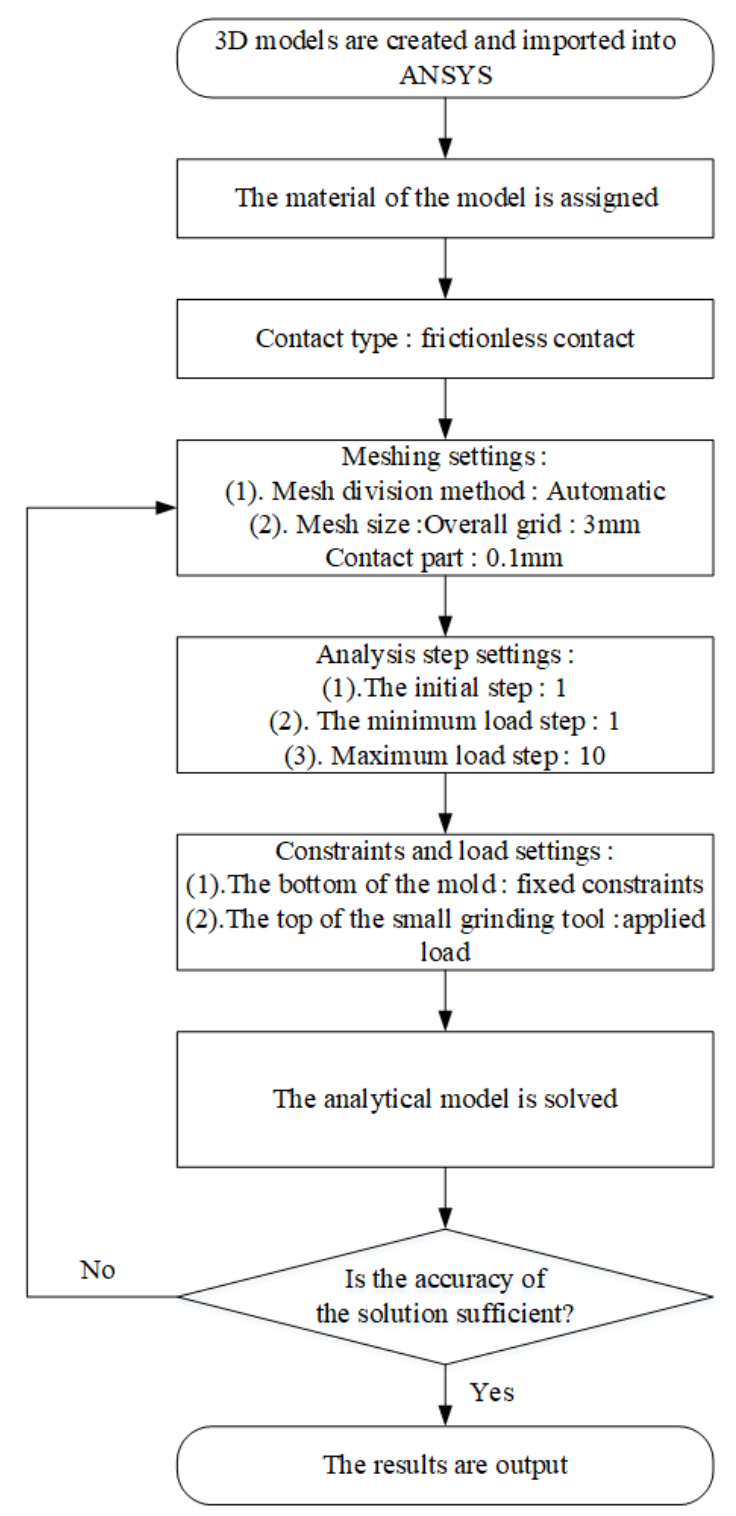

Fig. 2 Simulation flow chart

Tab. 2 Material parameter

\begin{tabular}{cccc}
\hline Material & $\begin{array}{c}\text { Elastic } \\
\text { Modulus } /(\mathrm{GPa})\end{array}$ & $\begin{array}{c}\text { Poisson's } \\
\text { ratio }\end{array}$ & Density $/\left(\mathrm{kg} / \mathrm{m}^{3}\right)$ \\
\hline $\begin{array}{c}\text { Workpiece } \\
(\mathrm{Q} 235)\end{array}$ & 212 & 0.3 & 7860 \\
\hline $\begin{array}{c}\text { Polishing } \\
\text { tool }\end{array}$ & 8.3 & 0.28 & 1150 \\
\hline
\end{tabular}

Three sets of displacement loads were applied to the small polishing tool, and the specific load parameters are shown in Tab. 1. The mold is pressed by the small polishing tool, and the contact starts from the center of the tool. The contact length is related to the applied load and the curvature of the mold. The shape of contact between the tool and the mold is elliptical, the contact profile is shown in Fig. 3(a). The contact stress diagram is shown in Fig. 3(b).

According to the Hertzian contact theory [14], the elastic plane touches the convex surface and generates an elliptical contact surface, the simulated stress cloud is close to the Hertzian Theory. The accuracy of the simulation results is proven. 


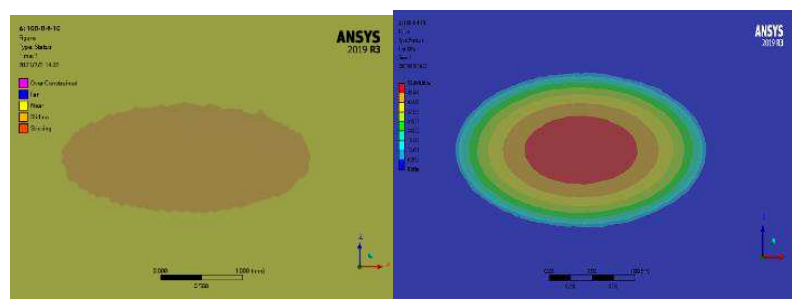

(a)contact area

(b) contact stress

Fig. 3 Contact area and contact stress of group 1

Three groups of displacement loads were applied to the small polishing tool for the comparison of the polishing force. The polishing force of the CDCP method in different positions of the mold is shown in Tab. 3. As the curvature of the mold surface increases from 0.005 to 0.020 , the first group of simulated polishing force decreases from $100 \mathrm{~N}$ to $69.21 \mathrm{~N}$. The second group of simulation polishing force was reduced from $150 \mathrm{~N}$ to $103.57 \mathrm{~N}$. The polishing force of the third group simulation was diminished from $200 \mathrm{~N}$ to $138 \mathrm{~N}$. The graph of polishing force variation of CDCP approach is displayed in the Fig.4.

From the simulation outcome, the higher the curvature, the greater the fluctuation of the polishing force of the CDCP. In the meantime, the contact area and contact stress between the small polishing tool and the mold would be affected.

Tab. 3 Force of CDCP $(N)$

\begin{tabular}{cccccc}
\hline $\begin{array}{c}\text { Position } \\
(\mathrm{mm})\end{array}$ & 0 & 25 & 50 & 75 & 80 \\
\hline Curvature & 0.005 & 0.006 & 0.007 & 0.011 & 0.020 \\
\hline Group 1 & 100.00 & 97.29 & 88.95 & 73.57 & 69.21 \\
\hline Group 2 & 150.00 & 146.21 & 133.32 & 110.01 & 103.57 \\
\hline Group 3 & 200.00 & 194.91 & 177.69 & 146.53 & 138.00 \\
\hline
\end{tabular}

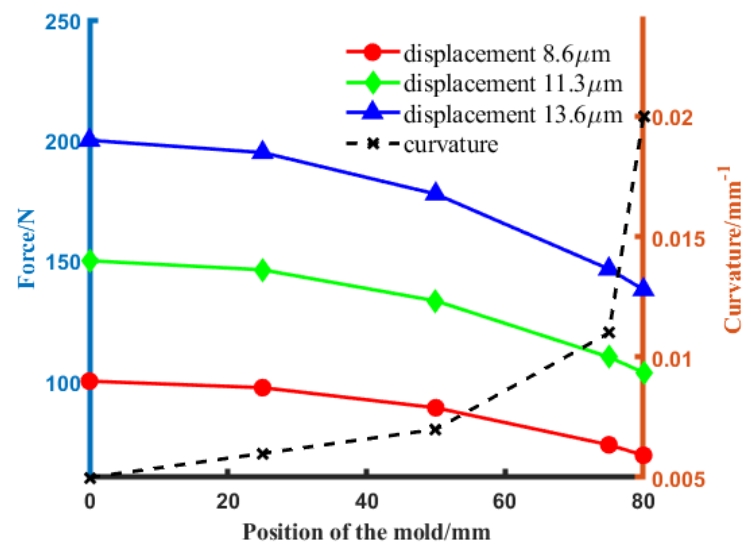

Fig. 4 Curve of the force of CDCP

The contact region between the small polishing tool and the mold is shown in Tab. 4, and the contact area decreased with the growth of curvature under the identical displacement load.

The relative contact area of the first group of CDCP simulations was diminished by $40.94 \%$, the second group was reduced by $46.68 \%$, the third group was declined by $47.15 \%$. The trend of contact 
area variations is shown in Fig. 5 (a).The contact stresses for the CDCP simulation are illustrated in Tab. 5. Under the same displacement load, the mold curvature raises and the contact stress increases. The contact stress in the first group increased by $30.28 \%$. The contact stress in the second group has grown by $31.72 \%$. The contact stress in the third group grows by $31.73 \%$. The trend of contact stress is demonstrated in Fig. 5 (b), where the contact stress increases and the material removal effect on the surface are enhanced.

In this section, the effect of the curvature of the mold under the CDCP method on the force varying during the polishing process is evaluated by simulation. The results suggest that the curvature of the mold rises, the force fluctuation increases, and the contact is more uniform. Therefore, based on the above research, a constant force control polishing (CFCP) method is suggested, and the influences of mold curvature on contact area and contact stress are investigated through simulation.

Tab. 4 Contact area of CDCP $\left(\mathrm{mm}^{2}\right)$

\begin{tabular}{cccccc}
\hline Position $(\mathrm{mm})$ & 0 & 25 & 50 & 75 & 80 \\
\hline curvature & 0.005 & 0.006 & 0.007 & 0.011 & 0.020 \\
\hline Group 1 & 2.76 & 2.62 & 2.27 & 1.63 & 1.45 \\
\hline Group 2 & 3.62 & 3.46 & 2.96 & 2.13 & 1.93 \\
\hline Group 3 & 4.39 & 4.20 & 3.58 & 2.57 & 2.32 \\
\hline Tab. 5 Contact stress of CDCP $(\mathrm{MPa})$ & \\
\hline Position(mm) & 0 & 25 & 50 & 75 & 80 \\
\hline curvature & 0.005 & 0.006 & 0.007 & 0.011 & 0.020 \\
\hline Group 1 & 55.87 & 56.96 & 60.65 & 69.56 & 72.79 \\
\hline Group 2 & 63.16 & 65.09 & 69.01 & 80.01 & 83.20 \\
\hline Group 3 & 69.49 & 71.59 & 75.89 & 87.97 & 91.54
\end{tabular}

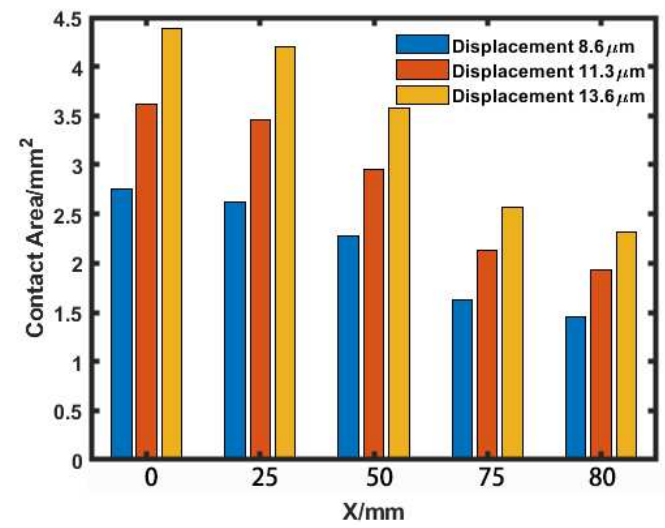

(a) contact area

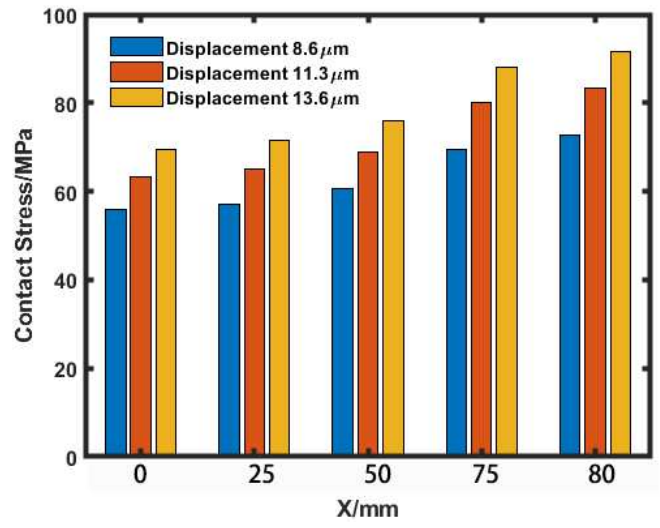

(b)contact stress

Fig. 5 Contact area and stress of constant displacement 


\subsection{Simulation analysis of constant force control polishing(CFCP)}

The same displacement load is applied to different positions of the mold in the robot CDCP method simulation. However, the displacement load is changed to force load in the CFCP simulation, which means the same force is applied at each position.

The rest of the constraints are also set identically. In the CFCP simulation, the force exerted on the small polishing tool in the first group is $100 \mathrm{~N}$, the force applying to the second group is $150 \mathrm{~N}$, the force imposed by the third group is $200 N$. The cause of the imposed load is shown in Tab. 1 .

The contact area outcomes of the CFCP simulation are shown in Tab. 7.The contact area of the first group of CFCP simulation is lowered by $32.72 \%$, The second group dropped by $32.04 \%$, The third group drops by $32.34 \%$. The contact area of each group of CFCP simulation is less varied than CDCP, and the detailed difference is shown in Tab. 6.

Tab. 7 Contact area of CFCP $\left(\mathrm{mm}^{2}\right)$

\begin{tabular}{cccccc}
\hline Position $(\mathrm{mm})$ & 0 & 25 & 50 & 75 & 80 \\
\hline Curvature & 0.005 & 0.006 & 0.007 & 0.011 & 0.020 \\
\hline Group 1 & 2.75 & 2.66 & 2.36 & 1.98 & 1.85 \\
\hline Group 2 & 3.62 & 3.50 & 3.11 & 2.60 & 2.46 \\
\hline Group 3 & 4.39 & 4.24 & 3.78 & 3.17 & 2.97 \\
\hline Experiment & CDCP & CFCP & Difference \\
\hline Number & $40.94 \%$ & $32.72 \%$ & $8.22 \%$ \\
\hline Group 1 & $46.68 \%$ & $32.04 \%$ & $14.64 \%$ \\
\hline Group 2 & $47.15 \%$ & $32.34 \%$ & $14.81 \%$ \\
\hline Group 3 & 47 area decrease ratio & \\
\hline
\end{tabular}

As the curvature of the mold increases, the contact stress rises. The increment of contact stress is higher of the CFCP method than CDCP. Greater contact stress represents more efficient material removal. Three groups of CFCP simulation contact stress results are indicated in Tab. 9.Under the same conditions, the contact stress of CFCP is larger than that of CDCP, which indicates that the material removal of the CFCP method is more complete.

The rates of increase of contact stress for both CFCP and CDCP methods are shown in Tab. 10. The trend of contact area and contact stress at various position of the mold is illustrated in Fig. 6.

Tab. 9 Contact stress of CFCP $(M P a)$ 


\begin{tabular}{cccccc}
\hline Position $(\mathrm{mm})$ & 0 & 25 & 50 & 75 & 80 \\
\hline Curvature & 0.005 & 0.006 & 0.007 & 0.011 & 0.020 \\
\hline Group 1 & 55.84 & 57.33 & 62.23 & 77.11 & 82.17 \\
\hline Group 2 & 63.13 & 65.47 & 70.82 & 88.65 & 94.02 \\
\hline Group 3 & 69.44 & 72.00 & 77.88 & 97.47 & 103.43 \\
\hline
\end{tabular}

Tab. 10 Contact stress increase ratio

\begin{tabular}{cccc}
\hline $\begin{array}{c}\text { Experiment } \\
\text { Number }\end{array}$ & CDCP & CFCP & Difference \\
\hline Group 1 & $30.28 \%$ & $47.15 \%$ & $16.87 \%$ \\
\hline Group 2 & $31.72 \%$ & $48.93 \%$ & $17.21 \%$ \\
\hline Group 3 & $31.73 \%$ & $48.94 \%$ & $17.21 \%$ \\
\hline
\end{tabular}

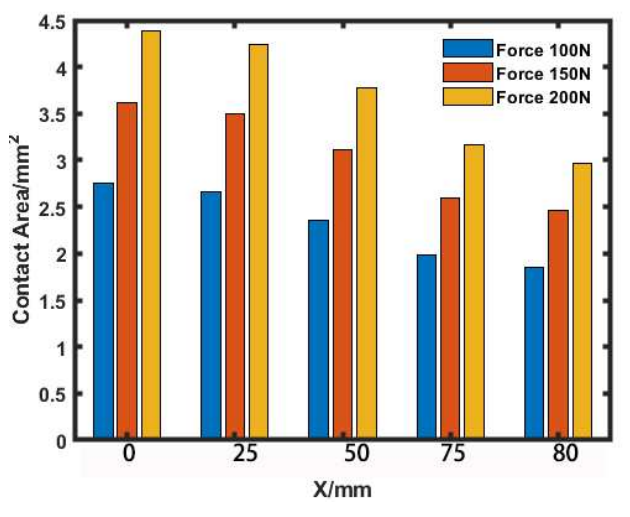

(a) contact area

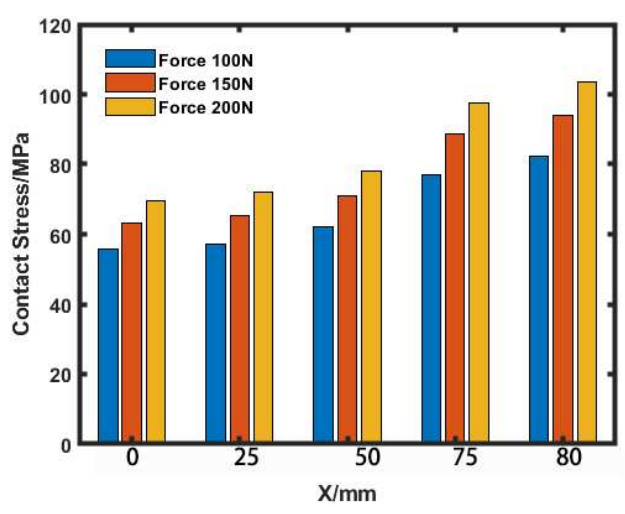

(b) contact stress

Fig. 6 Contact area and contact stress of constant force

From the simulation results, it can be seen that the mold is applied the same displacement load, the polishing force decreases with the increase of the curvature of the mold, and the small polishing tool cannot maintain a stable polishing force. Less variation in contact area and larger stress in CFCP can effectively improve polishing efficiency. Therefore, CFCP method is used in the actual processing of molds, which is achieved by a position-based adaptive impedance control algorithm.

\section{Constant force control polishing (CFCP) method based on position-based adaptive} impedance control

\subsection{Principle of Constant Force Control}

The strategy of constant force control means that the force error of the sensor is passed back to the controller and the position of the robot arm in the coordinate system is acquired. The control torque of the joint based on the inverse solution of the robot dynamics equations are calculated so that the adaptation of the robot arm position and contact force is realized. The schematic diagram of constant force control by the position-based adaptive impedance control method is shown in Fig. 7. 


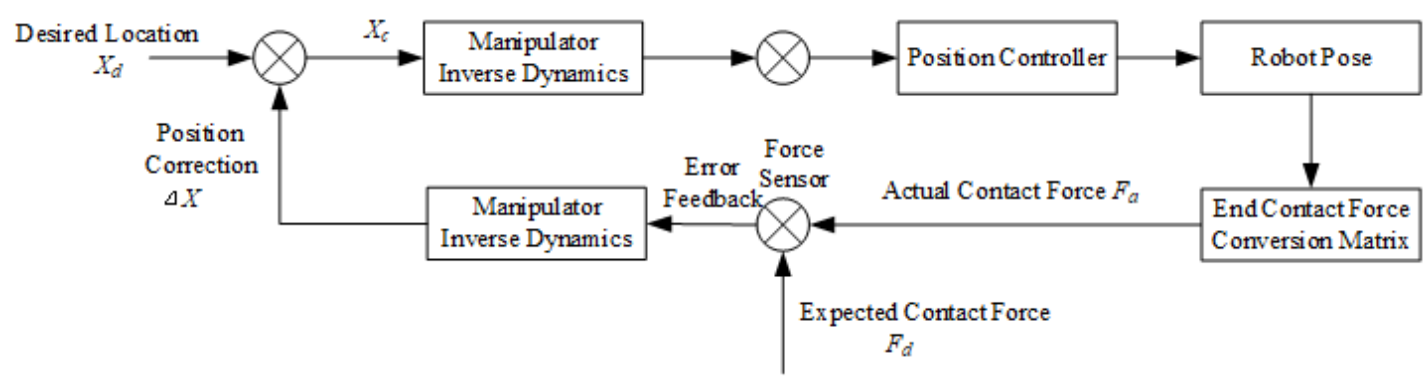

Fig. 7 Schematic diagram of constant force control

Impedance control is a functional relationship through the difference between the current state and the desired state. The feedback force message is combined with the difference value and the appropriate coefficient matrix is calculated in the next step.

The fitted control force $F_{\text {ext }}$ is corrected for the purpose of impedance control, and the second-order relationship between the fitted external force $F_{d}$ and the position offset $e$ is built as follows.

$$
M_{d} \ddot{e}+B_{d} \dot{e}+K_{d} e=F_{\text {ext }}
$$

Where $M_{d}$ is the target inertia matrix, $e$ is the position deviation, $B_{d}$ is the mark damping matrix, $K_{d}$ is the purpose stiffness matrix, and $F_{\text {ext }}$ is the external force. The correct control parameters are selected in the process to achieve the ideal target impedance.

\subsection{The implementation of position-based adaptive impedance control method}

The finishing of the mold by the robot is categorized into two stages: approaching and reaching the mold. By analyzing the robot control mode, the schematic block diagram of the position-based adaptive impedance control algorithm is proposed, and the particular steps are as follows:

(1) The contact process between the robot and the environment is divided into close and contact environment, corresponding to free and contact space.

(2) In free space, the initial position is gained by the controller, then the speed is modulated by the controller and transmitted to the impedance controller. The control form at that moment is

$$
f(\dot{x}, x, t)=-k_{v}\left(\dot{x}-\dot{x}_{d}\right)+k_{p}\left(-x+x_{d}\right)
$$

(3) The force corresponding to the speed is calculated by the impedance controller, the mold surface is reached by the small polishing tool at high speed.

(4) When reaching the contact space, the speed is modulated by impedance control and dynamic modulation to make it run at a low speed when it is close to the environment. The current mode of control is:

$$
f(\dot{x}, x, t)=-k_{v}(\dot{x})+k_{p}\left(-x+x_{d}\right)
$$

(5) When contacting the surface, only the impedance is used to control the force required to be applied to the small polishing tool.

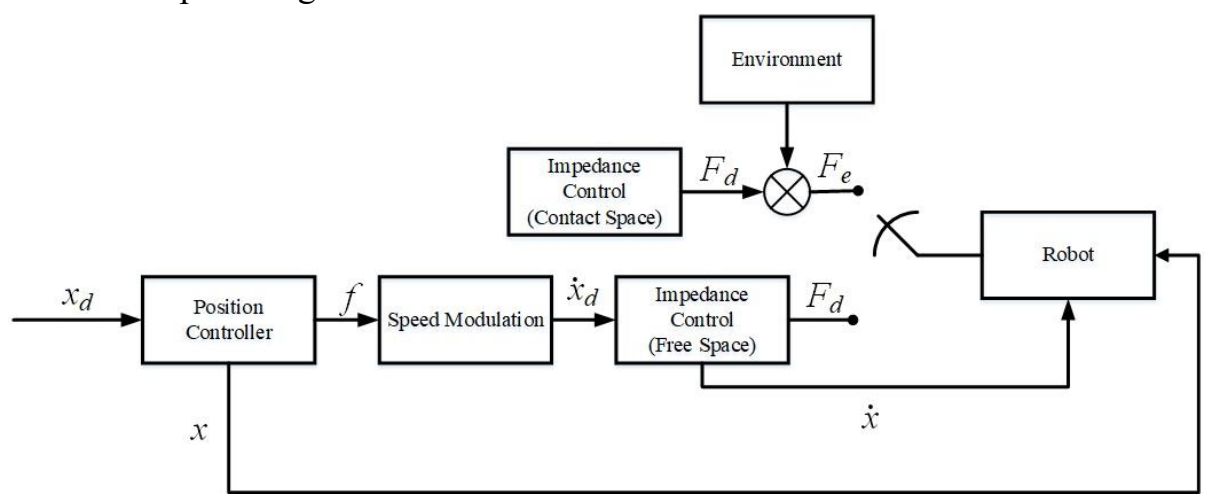

Fig. 8 Schematic diagram of adaptive impedance control based on position 
In the manipulated space, the robot is maintained constant polishing force by adaptive impedance control algorithm, while dynamically modulating with obstacle avoidance method [15]. CFCP has advantages such as fast force tracking and robustness compared with CDCP [15]. To verify the feasibility of the proposed method, the comparative experiments of CFCP and CDCP are conducted.

\section{Mold polishing experiment}

\section{1 experiment procedure}

The industry robot constant force control polishing bench mainly consists of a robot (installed with six-dimensional force/torque sensors and polishing tool), a working platform, and a water recycling cooling system, as depicted in Fig. 9.

The industrial robot is a six-axis manipulator arm, model KUKA-KR60, which is used to localize the small polishing tool through the motions of the arm. The 6-D force/torque sensor is an ATI Industrial Automation Delta IP60 model to acquire force data during the polishing process. The polishing tool is rotated by a servo motor, the motor power is $400 \mathrm{~W}$ and the maximum speed is $3000 \mathrm{r} / \mathrm{min}$. Three routes are selected on the mold surface for robotic polishing.

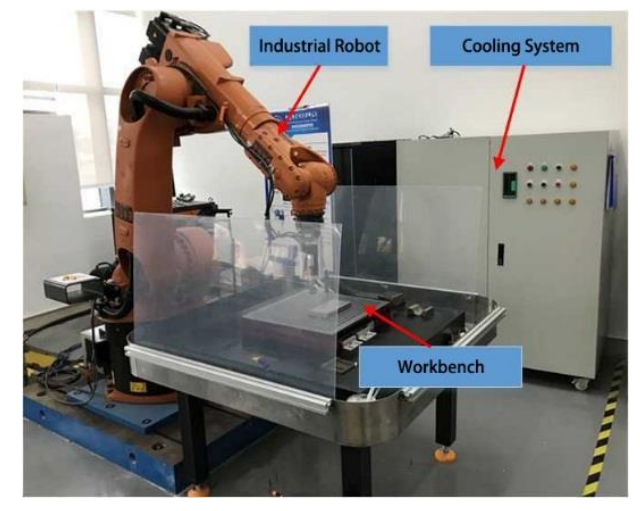

Fig. 9 Test platform of industrial robot

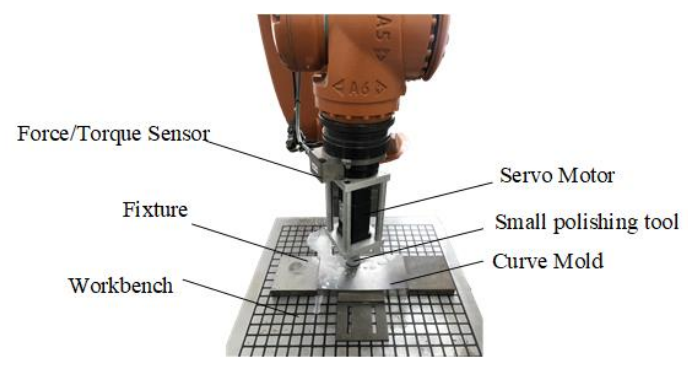

Fig. 10 Polishing process

On two molds with the same initial test conditions, the CFCP and CDCP experiments are carried out respectively. The robot polishing process is illustrated in Fig. 10.Mold material is Q235, mold size is $200 \times 100 \mathrm{~mm}$, the top is concave surface. The material of the small polishing tool is nylon and the size of the small tool is $\Phi 30 \times 25 \mathrm{~mm}$. The mass fraction of $\mathrm{Al}_{2} \mathrm{O}_{3}$ in the polishing fluid is $5 \%$. Small polishing tool rotational speed is $1000 \mathrm{r} / \mathrm{min}$ and feed velocity is $0.75 \mathrm{~mm} / \mathrm{s}$. The mold is attached by working magnetic platform and fixture, where the polishing force is set $-10 \mathrm{~N}$ in the CDCP and CFCP experiments (because the force sensor is subjected to a force in the negative direction of the Z-axis). The Japanese Mitutoyo SJ-210 stylus type surface roughness measuring instrument is used to evaluate the surface roughness of the mold after polishing.

3.2. Discussion of experiment results

3.2.1 Analysis of force wave in polishing process 
It can be observed that the machined surface having higher reflectivity after the three tracks were polished. The surface of the mold after CFCP of the small polishing tool is presented in Fig. 11.The polishing force data is captured by the force sensor and the variations of the three groups of forces are presented in Fig. 12..

The same trend of CDCP is observed in Fig. 12 of the polishing force graphs. Most of the polishing force is between $-15 N$ and $-5 N$, which varies very significantly. While significant force fluctuations are not observed in the CFCP approach, which is expected to be $-10 N$.

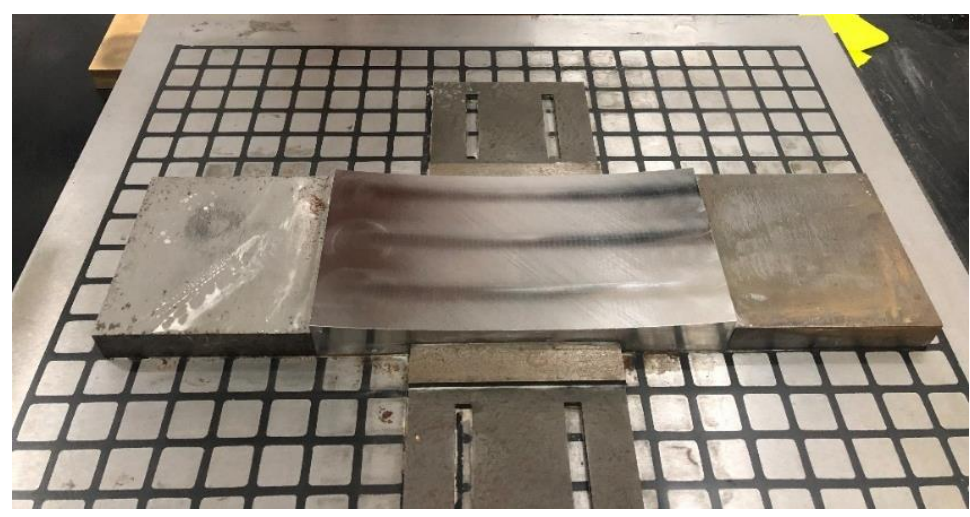

Fig. 11 The surface of the mold after finishing

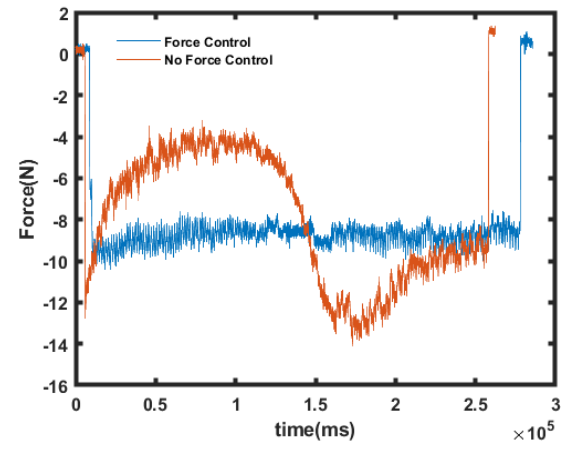

(a) The polishing force curve of the first path of the comparative test

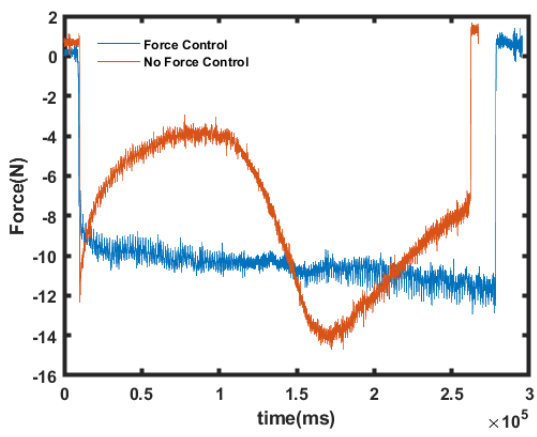

(b) The polishing force curve of the second path of the comparative test 


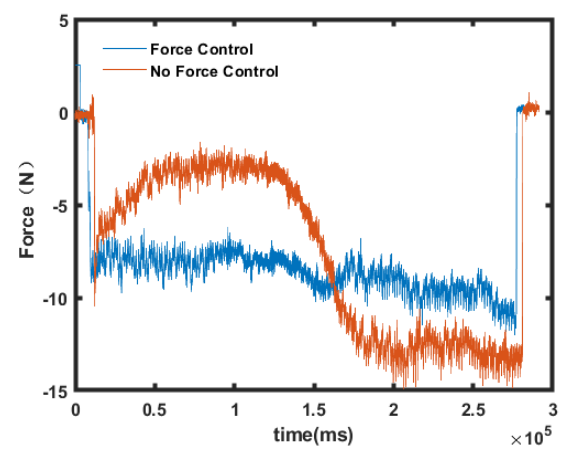

(c) The polishing force curve of the third path of the comparative test

Fig. 12 Curve of polishing force of robot on mold

To further analyze the variation of polishing force, the average and standard deviation of polishing force are calculated for each group of experiments. The difference between the CDCP method and desired force is $2.01 \mathrm{~N}, 1.97 \mathrm{~N}$ and $4.52 \mathrm{~N}$ respectively. Whereas, the gap between the CFCP method and the desired force is $1.49 \mathrm{~N}, 0.44 \mathrm{~N}$ and $0.74 \mathrm{~N}$ separately. The standard deviation of the polishing force of CFCP is lower than that of CDCP, which means that the fluctuation of force of the CFCP method is smaller. CFCP enable the finish surface to be uniform. The mean and standard deviation of the polishing force are provided in the Tab. 11 .

Tab. 11 Mean and standard deviation of polishing force

\begin{tabular}{ccccc}
\hline \multirow{2}{*}{$\begin{array}{c}\text { Mold } \\
\text { Path }\end{array}$} & \multicolumn{2}{c}{ Force Average } & \multicolumn{2}{c}{$\begin{array}{c}\text { Force Standard } \\
\text { Deviation }\end{array}$} \\
\cline { 2 - 5 } & CDCP & CFCP & CDCP & CFCP \\
\hline Path 1 & -7.99 & -8.51 & 3.58 & 0.65 \\
\hline Path 2 & -8.03 & -10.44 & 3.69 & 1.05 \\
\hline Path 3 & -14.52 & -9.26 & 5.57 & 1.04 \\
\hline
\end{tabular}

\subsubsection{Analysis of mold surface roughness}

Constant contact force is the key factor for polishing quality, so analysis is done by surface roughness. The results of the initial roughness and post-polishing roughness of the mold are presented in Tab. 12.The best surface quality of the mold is acquired by the CFCP trials, and the surface roughness is $0.595 \mu \mathrm{m}$.

The reduction percentages of CDCP are $25.78 \%, 47.13 \%, 48.30 \%$, and the reduction percentages of CFCP are $57.79 \%, 66.43 \%$, and $72.56 \%$ respectively. The specific surface roughness histogram is shown in Fig. 13. Because of the fluctuation of force in the polishing process, it causes uneven polishing on the surface of the mold. The surface quality is improved because of the constant polishing force by comparing two approaches.

Tab. 12 Surface roughness of the mold $(\mu m)$ 


\begin{tabular}{cccc}
\hline Control & Path 1 & Path 2 & Path 3 \\
\hline Initial surface & 1.912 & 2.13 & 2.168 \\
\hline CDCP & 1.419 & 1.126 & 1.120 \\
\hline CFCP & 0.807 & 0.715 & 0.595 \\
\hline
\end{tabular}

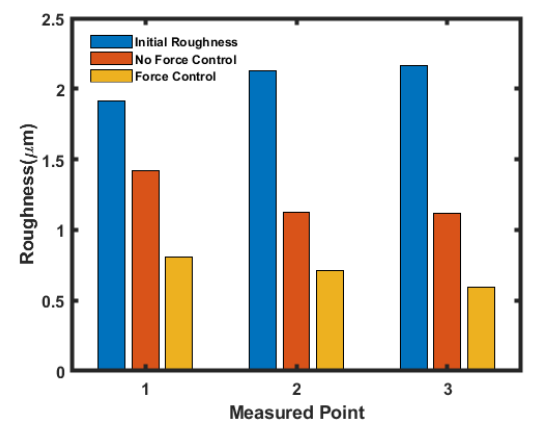

(a) Test results of the first route

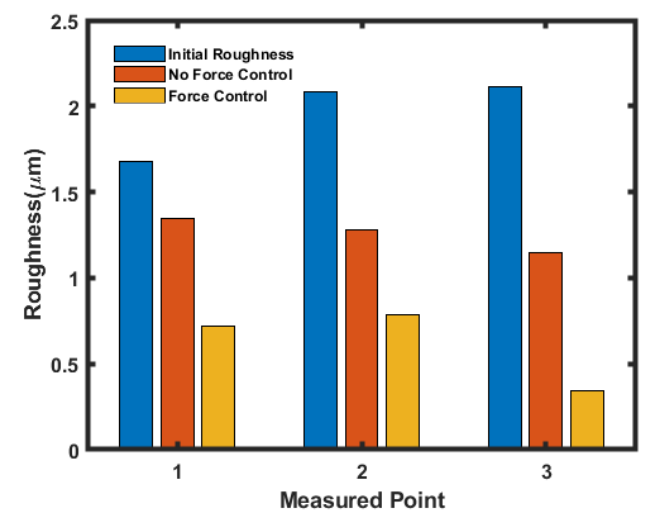

(b) Test results of the second route

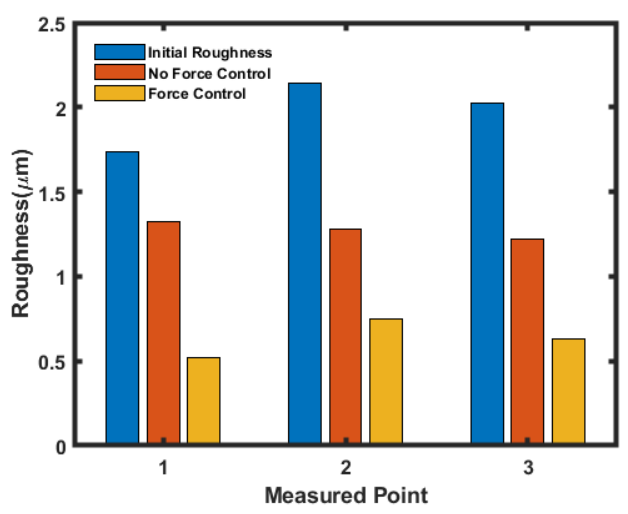

(c) Test results for the third route

Fig. 13 Histogram of surface roughness

\section{Conclusion}

The effect of mold curvature on the variation of polishing force with CDCP polishing method is investigated in this paper. The method of constant force control polishing is proposed for the disadvantage that CDCP cannot maintain constant force. The CFCP is achieved by a position-based adaptive impedance control algorithm and verified by experiments. The specific conclusions are as 
follows:

1. The curvature of the mold surface increases, the polishing force decreases. The polishing force cannot be kept stable in the CDCP method.

2. The curvature of the mold increases, the contact area between the small polishing tool and the mold decreases, the contact stress increases at the same load. The CFCP has greater contact area and contact stress than CDCP, indicating that CFCP is more adequately contacted and more efficient at the same curvature. The CFCP force is closer to the desired value than CDCP. The mean standard deviation of CDCP is 4.28 , and the standard deviation of CFCP is 0.91 .

3. The average reduction in roughness for the CFCP method is $65.59 \%$, the average reduction in roughness for the CDCP method is $40.42 \%$ on the same initial surface.

The effectiveness of the mold constant force polishing method based on position-based adaptive impedance control is proven by the experiment, which provides technical support to improve the efficiency of mold processing. 


\section{Acknowledgments}

The authors would like to thank Beijing Key Laboratory of Advanced Manufacturing Technology, Laboratory of Robotics and Intelligent Systems (CASQuanzhou) for the experimental support. The authors would also like to acknowledge the editors and the anonymous referees for their insightful comments.

\section{Ethical approval}

This material is the author's own original work, which has not been previously published elsewhere.

\section{Consent to participate}

Not applicable.

\section{Consent to publish}

The authors have read and agreed to publish the version of the manuscript.

\section{Author's contribution}

Guangsheng Chang: methodology, simulation analysis, writing-original draft. Yinhui Xie and Jinxing Yang: date curation, validation. Yong Yang: experiment operation. Ri Pan and Jun Li: writing-review and editing, resources.

\section{Funding}

This work was financially supported by the Nation Natural Science Foundation of China (Grant No.517750010) and Scientific and Technological Project of Quanzhou (No.2020C071).

\section{Conflict of interest}

The authors declare that they have no conflict of interest.

\section{Availability of data and material}

The experimental and simulation date is transparency. 


\section{References}

[1] Dahu Zhu,Xiaohu Xu,Zeyuan Yang,Kejia Zhuang,Sijie Yan,Han Ding. Analysis and assessment of robotic belt grinding mechanisms by force modeling and force control experiments[J]. Tribology International,2018,120.

[2] Ding Bingxiao,Zhao Jiyu,Li Yangmin. Design of a spatial constant-force end-effector for polishing/deburring operations[J]. The International Journal of Advanced Manufacturing Technology,2021,116(11-12):

[3] Kim Uikyum,Lee Dong Hyuk,Kim Yong Bum,Seok Dong Yeop,Choi Hyouk Ryeol. A Novel Six-Axis Force/Torque Sensor for Robotic Applications[J]. IEEE/ASME Transactions on Mechatronics,2017,22(3):

[4] J.H Ahn,Y.F Shen,H.Y Kim,H.D Jeong,K.K Cho. Development of a sensor information integrated expert system for optimizing die polishing[J]. Robotics and Computer Integrated Manufacturing,2001,17(4):

[5] H Huang,Z.M Gong,X.Q Chen,L Zhou. Robotic grinding and polishing for turbine-vane overhaul[J]. Journal of Materials Processing Tech.,2002,127(2):

[6] Abd El Khalick Mohammad,Jie Hong,Danwei Wang. Design of a force-controlled end-effector with lowinertia effect for robotic polishing using macro-mini robot approach[J]. Robotics and Computer Integrated Manufacturing,2018,49:

[7] Fan Chen,Huan Zhao,Dingwei Li,Lin Chen,Chao Tan,Han Ding. Contact force control and vibration suppression in robotic polishing with a smart end effector[J]. Robotics and Computer-Integrated Manufacturing,2019,57:

[8] Jie Tang,Tao Wang,Zhi Qi Yan,Li Wen Wang. Design and Analysis of the End-Effector of the Flexible Polishing Robot[J]. Key Engineering Materials,2016,4283(693-693):

[9] DUAN X L,HU J K,FANG J K et al. Research on grinding force fluctuation suppression methods of rail grinding train [J].Journal of Railway Science and Engineering 2014,11(02):125-130(in Chinese)

[10] Fengjie Tian,Zhenguo Li,Chong Lv,Guangbao Liu. Polishing pressure investigations of robot automatic polishing on curved surfaces[J]. The International Journal of Advanced Manufacturing Technology,2016,87(14).

[11] Dong Jinlong,Shi Jiashun,Liu Cong,Yu Tianbiao. Research of Pneumatic Polishing Force Control System Based on High Speed On/off with PWM Controlling[J]. Robotics and Computer-Integrated Manufacturing,2021,70.

[12] Ding Yufeng,Min Xinpu,Fu Weiwei,Liang Zilong. Research and application on force control of industrial robot polishing concave curved surfaces[J]. Proceedings of the Institution of Mechanical Engineers,2019,233

[13] SU C F,AI Y T,LOU X B. Study on contact stiffness factor selection method in contact nonlinear simulation[J].Journal of Shenyang Institute of Aeronautical Engineering 2009,26(03):5-9(in Chinese)

[14] J.A. Greenwood. Analysis of elliptical Hertzian contacts[J]. Tribology International,1997,30(3).

[15] FENG M Q,ZHU W B,Constant force grinding method based on dynamic system and impedance control[J],Information Technology 2020,44(08):7-11(in Chinese)

[16] Hamid Sadeghian,Fanny Ficuciello,Luigi Villani,Mehdi Keshmiri. Global Impedance Control of Dual-Arm Manipulation for Safe Interaction[J]. IFAC Proceedings Volumes,2012,45(22): 